\title{
Surdez Congénita ou Precocemente Adquirida: Do Rastreio ao Seguimento, um Retrato de Portugal
}

\author{
Congenital or Early Acquired Deafness: An Overview of \\ the Portuguese Situation, from Diagnosis to Follow-Up
}

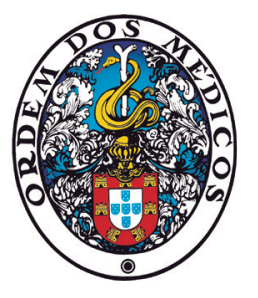

\author{
Catarina OLIVEIRA $\triangle^{1}$, Marta MACHADO², Raquel ZENHA ${ }^{1}$, Luísa AZEVEDO ${ }^{3}$, Luísa MONTEIRO ${ }^{4}$, Adelaide BICHO ${ }^{1}$ \\ Acta Med Port 2019 Dec;32(12):767-775 - https://doi.org/10.20344/amp.11880
}

\section{RESUMO}

Introdução: A surdez congénita ou precocemente adquirida afeta 1 a 3 por cada 1000 recém-nascidos sem fatores de risco e 20 a 40/1000 com fatores de risco. O rastreio auditivo neonatal universal permite a sua identificação precoce. As crianças com surdez congénita/precocemente adquirida têm uma maior prevalência de outras patologias, especialmente oftalmológicas e do neurodesenvolvimento, tendo pelo menos $30 \%$ a $40 \%$ uma comorbilidade associada.

Material e Métodos: Realizámos um estudo transversal, multicêntrico onde participaram $83 \%(n=30)$ dos hospitais/maternidades do Serviço Nacional de Saúde.

Resultados: Todos os hospitais/maternidades inquiridos realizam, por rotina, o rastreio auditivo neonatal universal a todos os recém-nascidos antes da alta; $63 \%$ encaminham para Otorrinolaringologia crianças com fatores de risco de surdez. Todas as crianças com surdez congénita/precocemente adquirida são encaminhadas para Pediatria em 23\% hospitais/maternidades. Em 23 hospitais/maternidades todas as crianças com surdez congénita/precocemente adquirida são encaminhadas para: Terapia da Fala em $44 \%$ hospitais/ maternidades; Oftalmologia em 17\% hospitais/maternidades; Sistema Nacional de Intervenção Precoce na Infância (SNIPI) em 30\% hospitais/maternidades; referenciação para Genética de todas as crianças com surdez congénita/ precocemente adquirida, sem causa identificada, em 22\% hospitais/maternidades. O número de diagnósticos de surdez nos anos de 2014 e 2015 foi de 2,5 e 1,5 por cada 1000 recém-nascidos, respetivamente, em 15 dos hospitais/maternidades.

Discussão: O rastreio auditivo neonatal universal parece estar amplamente difundido no Serviço Nacional de Saúde. O número de crianças com SC/SPA tal como a percentagem dos diferentes tipos de surdez diagnosticados, foram idênticos aos encontrados noutros estudos e mostra a indiscutível importância do rastreio. A avaliação/acompanhamento destas crianças por outras especialidades, além da Otorrinolaringologia, mostrou-se heterogéneo nas diferentes entidades de saúde e revelou que nem todas as crianças com fatores de risco de surdez realizam o seguimento aconselhado pelas normas existentes.

Conclusão: Os resultados mostram que Portugal realizou um percurso importante no âmbito do rastreio e seguimento das crianças com SC/SPA. Importa, com o fim último da melhoria continua da prestação de cuidados a estas crianças, refletir sobre o envolvimento de outras especialidades, além da Otorrinolaringologia, tal como do Sistema Nacional de Intervenção Precoce na Infância no seguimento destas crianças.

Palavras-chave: Portugal; Rastreio Neonatal; Surdez/congénita; Surdez/diagnóstico; Testes Auditivos

\section{ABSTRACT}

Introduction: Congenital deafness or early acquired deafness affects 1 to 3 out of 1000 newborns without risk factors and 20 to 40 out of 1000 newborns with risk factors. The universal newborn hearing screening enables its early identification. Children with congenital deafness/early acquired deafness have a higher prevalence of other conditions, especially ophthalmologic and neurodevelopmental ones, and at least $30 \%$ to $40 \%$ have at least one associated comorbidity.

Material and Methods: We carried out a cross-sectional, multicenter study in which $83 \%(n=30)$ of the hospitals/maternity hospitals of the National Health Service participated.

Results: All surveyed hospitals/maternity hospitals routinely performed universal newborn hearing screening to all newborns before discharge; $63 \%$ referred children with risk factors for hearing loss to Otorhinolaryngology. All children with congenital deafness/early acquired deafness are referred to: Pediatrics in $23 \%$ hospitals/maternity hospitals. In 23 hospitals/maternity hospitals, all children with congenital deafness/early acquired deafness are referred to: Speech Therapy in $44 \%$ hospitals/ maternity hospitals; Ophthalmology in $17 \%$ hospitals/maternity hospitals; National System of Early Intervention in Childhood in $30 \%$ hospitals/maternity hospitals; $22 \%$ of hospitals/maternity hospitals refer all children with congenital deafness/early acquired deafness, with no identified cause, to Clinical Genetics clinics. The number of diagnoses of deafness in the years 2014 and 2015 was 2.5 and 1.5 per 1000 newborns, respectively, in 15 hospitals/maternity hospitals.

Discussion: Awareness of universal newborn hearing screening seems to be widely spread in the National Health Service. The number of children with SC / SPA, as well as the percentage of different types of deafness diagnosed, were identical to those found in other studies and shows its importance. The assessment / follow-up of these children by specialties other than the otolaryngology was heterogeneous in different health entities and revealed that not all children with risk factors for deafness follow up advised by existing standards. Conclusion: Results show that Portugal made an important path in the screening and follow-up of children with SC / SPA. It is important, with the ultimate aim of continually improving the care of these children, to reflect on the involvement of specialties other than otolaryngology, such as the National Early Childhood Intervention System in the follow-up of these children.

Keywords: Deafness/congenital; Deafness/diagnosis; Hearing Tests; Neonatal Screening; Portugal

\footnotetext{
1. Serviço de Pediatria. Centro Hospitalar do Baixo Vouga. Aveiro. Portugal.

2. Serviço de Pediatria. Hospital Pediátrico de Coimbra. Coimbra. Portugal.

3. Serviço de Otorrinolaringologia. Centro Hospitalar do Baixo Vouga. Aveiro. Portugal.

4. Serviço de Otorrinolaringologia. Hospital Lusíadas. Lisboa. Portugal.

$\triangle$ Autor correspondente: Catarina Sousa Oliveira. catarina.a.sousa.oliveira@gmail.com

Recebido: 01 de fevereiro de 2019 - Aceite: 12 de junho de 2019 | Copyright @ Ordem dos Médicos 2019
} 


\section{INTRODUÇÃO}

A surdez é uma das patologias mais prevalentes do período neonatal, afeta 1 a 3 por cada 1000 recém-nascidos (RN) sem fatores de risco e 20 a 40 por cada 1000 $\mathrm{RN}$ com fatores de risco para surdez. ${ }^{1-6}$ Detetada neste período, pode ser congénita ou precocemente adquirida. Etiologicamente a surdez congénita (SC) divide-se entre genética ( $60 \%$ ) e adquirida (in utero) ${ }^{7,8} \mathrm{~A}$ genética em $60 \%$ dos casos é não sindrómica; a adquirida é frequentemente de etiologia infeciosa, especialmente por agentes do grupo TORCH (toxoplasmose, rubéola, citomegalovírus, herpes, outras doenças), onde se destaca o citomegalovírus. ${ }^{7-9} \mathrm{~A}$ surdez precocemente adquirida (SPA) associa-se à prematuridade e num menor número de casos à ação de substâncias ototóxicas (p.ex. aminoglicosídeos; hiperbilirrubinémia com necessidade de exsanguíneo-transfusão), asfixia perinatal grave ou meningite bacteriana. ${ }^{7,8,10}$

A apresentação clínica da SC/SPA pode ser imediata, progressiva ou tardia, conforme a etiologia. ${ }^{11,12} \mathrm{O}$ rastreio auditivo neonatal universal (RANU) permite identificar os possíveis casos de surdez que são posteriormente confirmados (ou não) por Otorrinolaringologia (ORL). No caso específico de RN com fatores de risco de surdez (Tabela 1) o seu seguimento deve ser mantido até à aquisição de linguagem, mesmo que o RANU seja negativo. ${ }^{13}$

A realização do rastreio no período neonatal permite que a reabilitação auditiva se inicie antes dos seis meses de idade, período chave em que as sinapses entre o córtex auditivo e o centro da linguagem se formam. ${ }^{14,15} \mathrm{~A}$ intervenção iniciada até esta idade leva a que o desenvolvimento da linguagem da criança com SC/SPA, sem outras comorbilidades, seja similar ao daquela sem surdez, com a mesma capacidade cognitiva. ${ }^{16,17}$ Adicionalmente, porque a prevalência de outras patologias nas crianças com SC/SPA é superior à das crianças sem surdez (cerca de 30\% a 40\% têm pelo menos uma comorbilidade associada), é importante o seu acompanhamento por uma equipa multidisciplinar capaz de as identificar e tratar. ${ }^{18-23}$ As comorbilidades mais frequentes são as patologias oculares, que em algumas séries chegam a atingir os $60 \%$ (p.ex. erros refratários, perturbação da visão binocular, ambliopia, estrabismo, perturbações visuais e cegueira). ${ }^{24,25} \mathrm{~A}$ avaliação oftalmológica destas crianças, por rotina, pode permitir diagnósticos e tratamentos mais precoces. ${ }^{7,26}$ As patologias associadas ao neurodesenvolvimento afetam cerca de $30 \%$ destas crianças (p.ex. perturbação da motricidade, perturbação do espectro do autismo, défice cognitivo, perturbação de hiperatividade e défice de atenção e dificuldades de aprendizagem). ${ }^{16,23,24,27} \mathrm{~A}$ sua prevalência é idêntica qualquer que seja o grau de défice auditivo (DA). ${ }^{21,23} \mathrm{~A}$ realização de testes padronizados para a sua deteção é de extrema importância. Sabe-se que a avaliação do desenvolvimento do lactente e da criança por um Pediatra, sem a realização desses testes, leva à omissão destes diagnósticos, em idade pré-escolar, numa percentagem que pode chegar aos $60 \% .{ }^{28-30} \mathrm{O}$ atraso do desenvolvimento da linguagem pode existir em mais de $50 \%$ destas crianças mesmo nos casos de surdez ligeira a moderada a beneficiarem de próteses auditivas. ${ }^{16,21}$ Estudos de caso-controlo têm revelado diagnósticos de atraso no desenvolvimento da linguagem, identificados por profissionais especializados, em $60 \%-70 \%$ de crianças com SC/SPA, onde se incluem crianças sem suspeita de atraso do desenvolvimento da linguagem. ${ }^{16,21}$ Em conclusão, a complexidade dos doentes com SC/SPA pode implicar não só a reabilitação auditiva — adaptação a próteses auditivas e em alguns casos a implantes cocleares, que será sempre o tratamento chave, mas também a intervenção em outros órgãos/sistemas. Adicionalmente, a reabilitação destes doentes não deve ficar circunscrita ao meio hospitalar, sendo o envolvimento da família e da comunidade educativa de extrema importância. ${ }^{16,31-33}$ Importa, por isso, recordar o Sistema Nacional de Intervenção Precoce na Infância (SNIPI), um projeto nacional, constituído por equipas multidisciplinares, que tem como objetivo apoiar as crianças com risco de atraso do desenvolvimento e respetiva família. Intervém de modo centrado na família nos vários âmbitos da reabilitação precoce, articulando as necessidades da criança com a comunidade escolar, serviços de saúde e de ação social. ${ }^{34}$

\section{RANU e intervenção precoce em Portugal}

A primeira recomendação europeia para a realização do RANU, o European Consensus Statement of Neonatal Hearing, data de $1998 .{ }^{35}$ Sete anos mais tarde é criado

Tabela 1 - Indicadores de risco para surdez no recém-nascido

\section{Fatores de risco}

História familiar de deficiência auditiva congénita

Infeção congénita (sífilis, toxoplasmose, rubéola, citomegalovírus e herpes)

Anomalias craniofaciais (malformações de pavilhão auricular, meato acústico externo, ausência de filtrum nasal, implantação baixa da raiz do cabelo)

Peso ao nascimento inferior a $1500 \mathrm{~g}$

Hiperbilirrubinémia (níveis séricos indicativos de exsanguineo-transfusão)

Medicação ototóxica por mais de 5 dias (aminoglicosídeos ou outros, associados ou não aos diuréticos de ansa)

Meningite bacteriana

Índice de Apgar de 0 - 4 no primeiro minuto ou 0 - 6 no quinto minuto

Ventilação mecânica por período igual ou superior a 5 dias

Sinais ou síndromes associadas à deficiência auditiva de condução ou neurossensorial 
em Portugal o Grupo de Rastreio e Intervenção da Surdez Infantil (GRISI). No ano de 2007 o GRISI publica na Acta Pediátrica Portuguesa "Recomendações para o Rastreio Auditivo Neonatal Universal"36 onde é aconselhado que: o RANU se realize até ao final do primeiro mês de vida a todos os RN; a avaliação audiológica por ORL dos lactentes com défice auditivo (DA) detetado pelo RANU seja efetuada até aos três meses de idade; a intervenção se inicie antes dos seis meses; o seguimento por ORL das crianças com fatores de risco para surdez se mantenha até cerca dos três anos de idade. Posteriormente a esta publicação vários profissionais de saúde, ligados à ORL e à Pediatria/Neonatologia iniciaram o RANU nos seus serviços. Em dezembro de 2015 foi publicada uma norma de orientação clínica (NOC) da Direção Geral de Saúde (DGS) sobre o rastreio e tratamento da surdez com implantes cocleares em idade pediátrica, atualizada em julho de 2017. ${ }^{13}$ A NOC corrobora a publicação do GRISI e especifica o tratamento e o seguimento das crianças com DA mais graves com necessidade de colocação de implantes cocleares. ${ }^{13}$ As crianças com DA menos graves não são contempladas nesta NOC. As recomendações do GRISI pelo seu carácter mais universal, abrangem estas crianças. No entanto, no que diz respeito ao seguimento por outras especialidades além da ORL, as recomendações são muito genéricas: "A avaliação e o seguimento de uma criança com perda auditiva deverão ser realizados por uma equipa multidisciplinar que inclua pediatras, otorrinolaringologistas, audiologistas, enfermeiros, terapeutas da fala, psicólogos e outros." Não é referida a avaliação por parte de Oftalmologia, não é indicada em que idades estas crianças devem ser referenciadas e não esclarece se esta avaliação multidisciplinar deve ser realizada por todas aquelas especialidades e se a todas as crianças com DA, mesmo que os défices sejam ligeiros e as crianças sejam saudáveis.

Até à data atual, o único estudo do nosso conhecimento, de abrangência nacional, que aborda a taxa de implementação do RANU e o número de diagnósticos de surdez realizados através deste rastreio, é a tese de mestrado "Rastreio Auditivo Neonatal Universal em Portugal: Ponto da Situação" da autora Ana Rita Ferreira Canas dos Santos. ${ }^{37}$ Não encontrámos nenhum estudo, de âmbito nacional, que analisasse o modo como as crianças com SC/SPA são acompanhadas por parte de outras especialidades além da ORL.

\section{Objetivos}

1. Quantificar os hospitais com maternidade e maternidades do Serviço Nacional de Saúde (SNS) que realizam o RANU por rotina antes da alta hospitalar do RN;

2. Identificar o número de diagnósticos de SC/SPA, realizados no âmbito do RANU, por nados-vivos nascidos nos anos de 2014 e 2015 no SNS;

3. Descrever o seguimento realizado às crianças com fatores de risco de surdez. Descrever o seguimento realizado às crianças com surdez detetada pelo RANU no SNS por outras especialidades além de ORL;

4. Conhecer em que casos o SNIPI é ativado.

\section{MATERIAL E MÉTODOS}

Realizámos um estudo transversal, quantitativo e descritivo. Primeiro, fizemos o levantamento de todos os hospitais/ centros hospitalares/unidades locais de saúde existentes no SNS, à data da realização do estudo (setembro de 2016), recorrendo ao site governamental https://www.sns.gov.pt/ institucional/entidades-de-saude. Contactámos os respetivos hospitais/centros hospitalares/unidades locais de saúde e verificámos quais os que possuíam bloco de partos e maternidade. Foram incluídos no estudo 36 hospitais/centros hospitalares/unidades locais de saúde (a este conjunto de locais de saúde passamos a designar doravante apenas por entidades de saúde). Numa segunda fase do estudo, elaborámos um questionário composto por 13 perguntas de escoIha múltipla e quatro de resposta aberta, que incidiam sobre:

a) a metodologia utilizada no RANU, na orientação e no seguimento das crianças com fatores de risco de surdez e daquelas com diagnóstico de SC/SPA detetada através do RANU;

b) o número de diagnósticos de DA realizados através do RANU nos anos de 2014 e 2015.

Posteriormente, inserimos o questionário na plataforma digital SurveyMonkey® e enviámo-lo por correio eletrónico e por carta (nos casos em que não houve resposta ao correio eletrónico) a todos os diretores de serviço de ORL e de Pediatria de todas as entidades de saúde incluídas no estudo. Aceitámos respostas ao questionário até 31 de julho de 2017. Na última fase do estudo, inserimos os dados recoIhidos numa base de dados em Excel ${ }^{\circledR}$ e realizámos a sua análise nesse mesmo programa.

A análise das respostas obtidas teve em consideração os seguintes pressupostos:

- Quer o serviço de Pediatria quer de ORL, seja de um hospital nível $1 / 2$ ou 3 tem conhecimento se os RN nascidos na sua instituição realizam ou não o RANU antes da alta hospitalar e quais são os profissionais que o realizam;

- Nas maternidades e hospitais distritais nível 1/2, pode ser realizado apenas o RANU, podendo os $\mathrm{RN}$ que não passam na primeira ou segunda fase do rastreio ou que tenham fatores de risco de surdez ser encaminhados para hospital de referência. Nestes casos, apenas este último pode informar sobre o seguimento realizado a estas crianças. Neste sentido, os hospitais nível $1 / 2$ que responderam ao questionário foram contatados com o objetivo de se saber o tipo de seguimento que realizavam às crianças com alterações no RANU ou SC/SPA - seguimento na íntegra até colocação de implantes cocleares (inclusive ou exclusive) ou encaminhamento de todas estas crianças para hospital de referência;

- O serviço de Pediatria sabe na íntegra todo o seguimento realizado à criança com SC/SPA se a ele forem referenciadas por rotina todas estas crianças.

Partindo das condições acima referidas, os dados obtidos através das respostas ao questionário foram divididos 

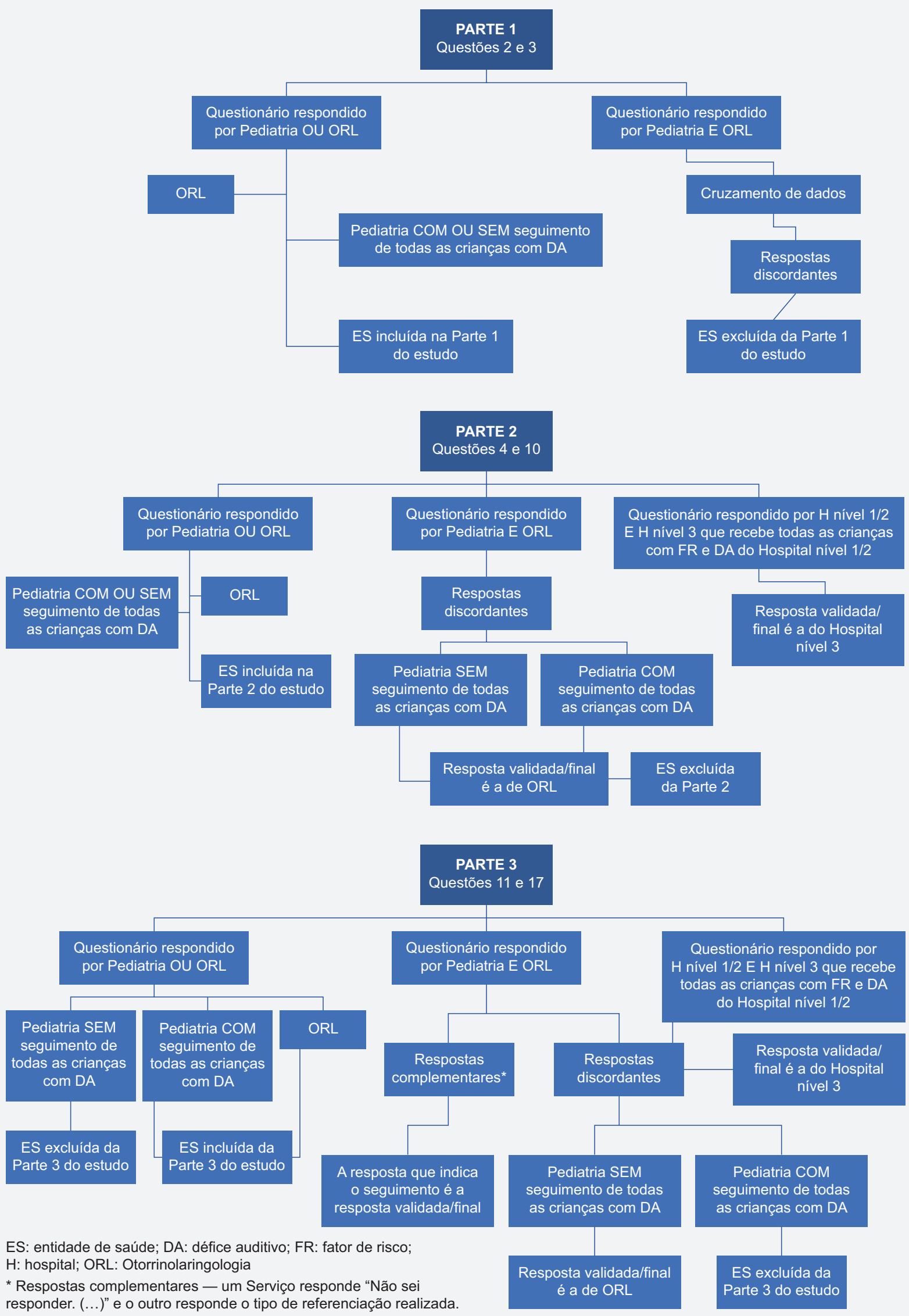

Figure 1 - Diagrama explicativo dos critérios utilizados para validação das respostas e inclusão no estudo 
em três partes. Parte 1 - perguntas 2 e 3 (realização por rotina do RANU antes da alta e quais os profissionais que o realizam); Parte 2 - perguntas 4 a 10 (número de nados vivos nascidos em 2014 e 2015 e respetivos diagnósticos de SC/SPA detetados através do RANU; seguimento realizado aos RN que não passam (REFERE) na primeira fase do rastreio e aos $\mathrm{RN}$ com fatores de risco de surdez); Parte 3 - questões 11 a 17 (seguimento das crianças com DA por outras especialidades além da ORL)

As respostas foram validadas como representativas da realidade da entidade de saúde, representada pela resposta dada pelo serviço de ORL e/ou Pediatria, da forma indicada no diagrama apresentado na Fig. 1

Este estudo contou com o apoio científico do GRISI, da Sociedade Portuguesa de Otorrinolaringologia e Cirurgia de Cabeça e Pescoço, e da Sociedade Portuguesa de Pediatria.

\section{RESULTADOS}

Do total de 36 entidades de saúde, 30 responderam ao questionário (taxa de resposta de $83 \%$ ). A resposta foi dada através do serviço de ORL em $37 \%$ dos casos, de Pediatria em $33 \%$ (onde se inclui uma maternidade) e por ambos os serviços (ORL e Pediatria) em $30 \%$ dos casos.

\section{Parte 1.}

\section{RANU}

Entidades de saúde representadas: 30 (nenhuma das entidades de saúde apresentou critério de exclusão).

- Todas realizam por rotina o RANU a todos os RN, antes da alta hospitalar.

- Na maioria (67\%) das entidades de saúde, o audiologista é o responsável pela realização do RANU; nos restantes casos, o rastreio é realizado por médico/enfermeiro e em algumas entidades em alguns dias por audiologista.

\section{Parte 2.}

2.1 Número de nados-vivos nascidos nos anos de 2014 e 2015

Das 30 entidades de saúde, 27 responderam à questão relativa ao número de nados-vivos nascidos no seu hospital/maternidade no ano de 2014 e 26 relativamente ao ano de 2015:

- Nados-vivos 2014 (27 entidades de saúde): 40196

- Nados-vivos 2015 (26 entidades de saúde): 40720

2.2 Número de RN com DA detetado através do RANU nos anos de 2014 e 2015 (Fig 2)

Entidades de saúde representadas: 15 (13 hospitais nível $1 / 2$ e dois hospitais nível 3)

\section{Ano 2014}

- O diagnóstico de SC/SPA foi realizado a um total de 64 crianças, 36\% ( $n=23)$ apresentavam hipoacusia moderada, 25\% $(n=16$,$) hipoacusia ligeira, 14\% (n=9)$ hipoacusia profunda, $12,5 \%(n=8)$ hipoacusia severa e $12,5 \%$ ( $n=8)$ DA total (cofose).

- O número total de nados-vivos nascidos nas 15 instituições de saúde foi de 25623 , pelo que o número de RN com DA, nesta amostra, foi de 2,5 por cada 1000 RN.

\section{Ano 2015}

- Foi realizado o diagnóstico de SC/SPA em 38 crianças, 34\% $(n=13)$ tinham hipoacusia moderada, $21 \%(n=8)$ hipoacusia ligeira, 21\% ( $n=8)$ hipoacusia severa, $13 \%$ $(n=5)$ hipoacusia profunda, $11 \%(n=4)$ DA total (cofose).

- O número de nados-vivos nas 15 instituições foi de 26056 pelo que o número RN com DA por cada $1000 \mathrm{RN}$, nesta amostra, foi de 1,5 por cada $1000 \mathrm{RN}$.

2.3 Orientação do RN REFERE na primeira fase do RANU e do RN com fatores de risco de surdez

Entidades de saúde representadas: 30 (nenhuma das entidades de saúde apresentou critério de exclusão).

- Todas encaminham as crianças que não passam na primeira fase do RANU para a segunda fase.

Número de RN com SC/SPA detetada através do RANU nos anos de 2014 e 2015

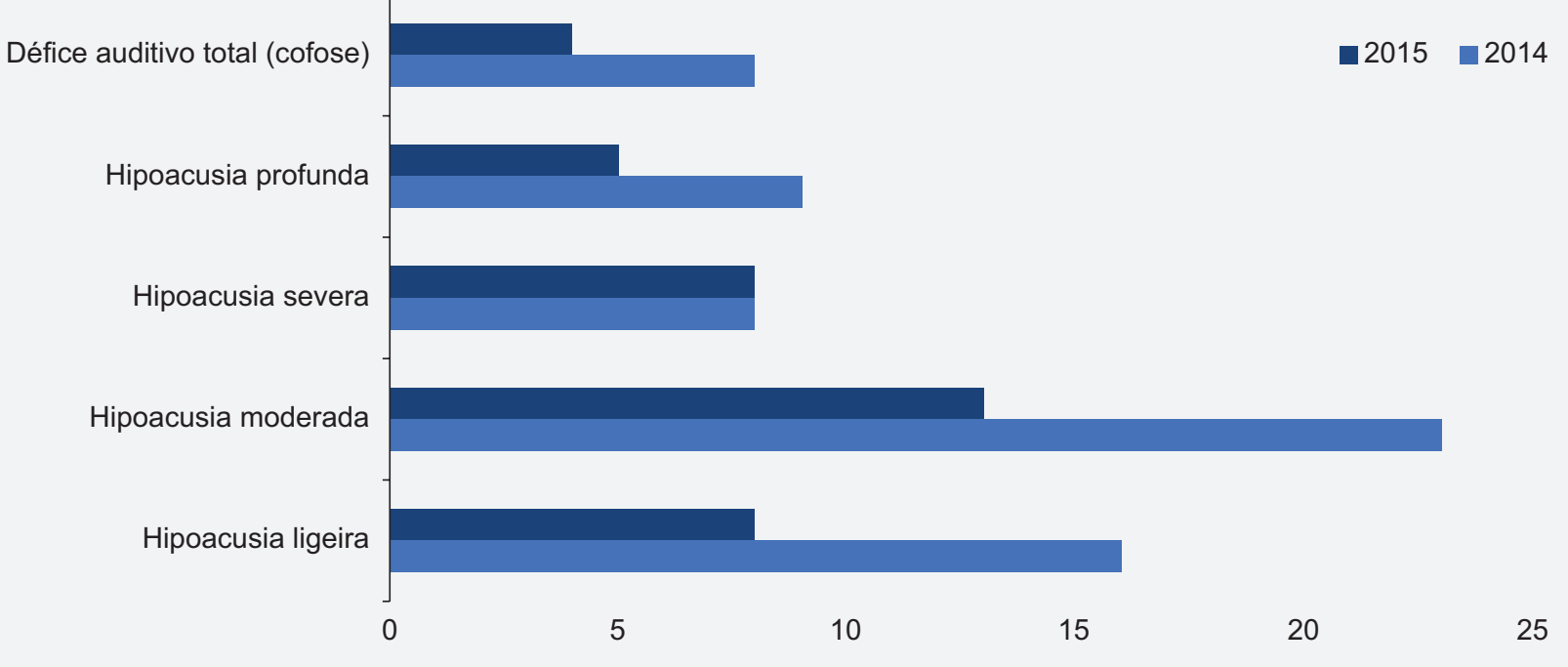

Figure 2 - Número de RN com DA detetado através do RANU nos anos de 2014 e 2015 
- Dezanove (63\%) encaminham para consulta de ORL as crianças com fatores de risco de surdez, mesmo que passem na primeira fase do RANU. Duas entidades de saúde dão alta para o médico de família. As restantes $(30 \% ; n=9)$ tomam diferentes atitudes: encaminham para consulta de Neonatologia ou Desenvolvimento ( $\mathrm{n}=$ 3 ); solicitam a realização de potenciais evocados auditivos do tronco cerebral $(n=5)$; uma entidade de saúde encaminha para consulta de ORL se o RN tiver como fatores de risco peso ao nascer $<1500 \mathrm{~g}$ ou $<32$ semanas de idade gestacional

2.3 Referenciação para Pediatria ou subespecialidade (Tabela 2)

Entidades de saúde representadas: 30 (nenhuma das entidades de saúde apresentou critério de exclusão).

\section{Parte 3}

3.1. Referenciação para Terapia da Fala, Oftalmologia Genética e SNIPI (Tabela 2)

Entidades de saúde representadas: 23 (dois hospitais nível 3 e 21 nível 1/2). Das 30 entidades de saúde foram excluídas sete, cujos questionários foram respondidos somente pelos serviços de Pediatria aos quais não eram referenciadas todas as crianças com SC/SPA).

\subsection{Serviço responsável pela ativação do SNIPI}

Entidades de saúde representadas: 23 (as mesmas do ponto 3.1)

- Em todas estas entidades de saúde, o serviço responsável pela ativação do SNIPI é sempre o de Pediatria.

3.3 Apoio para a integração da criança na comunidade quando o SNIPI não é ativado

Tabela 2 - Referenciação das crianças com SC/SPA para Pediatria/subespecialidade, Oftalmologia, Genética e SNIP

\begin{tabular}{|c|c|c|c|c|c|c|}
\hline \multicolumn{7}{|c|}{ Grau de diferenciação do hospital e do serviço de ORL } \\
\hline $\begin{array}{l}\text { Referenciação } \\
\text { ( } n=\text { ES } \\
\text { representadas) }\end{array}$ & $\begin{array}{l}\text { Crianças c/ SC/SPA } \\
\text { referenciadas }\end{array}$ & $\begin{array}{l}\text { H nível } 1 / 2 \\
\text { ORL A (n) }\end{array}$ & $\begin{array}{l}\text { H nível } 1 / 2 \\
\text { ORL } B(n)\end{array}$ & $\begin{array}{l}\text { H nível } 1 / 2 \\
\text { ORL C (n) }\end{array}$ & H nível 3 (n) & $\begin{array}{l}\text { Total } \\
\mathrm{n}(\%)\end{array}$ \\
\hline \multirow{3}{*}{$\begin{array}{l}\text { Pediatria/ } \\
\text { subespecialidade } \\
n=30\end{array}$} & Todas & & 4 & 3 & & $7(23 \%)$ \\
\hline & Nenhuma (por rotina) & 1 & 6 & 4 & $4 \dagger$ & $15(50 \%)$ \\
\hline & $\begin{array}{l}\text { Se DA de determinada } \\
\text { gravidade (qual) }\end{array}$ & & $\begin{array}{c}7 \\
\text { (6 DA } \geq \text { moderado; } \\
1 \mathrm{DA} \geq \text { severo) }\end{array}$ & & $\begin{array}{c}1 \\
(\mathrm{DA} \geq \text { moderado })\end{array}$ & $8(27 \%)$ \\
\hline Total & & & & & & 30 \\
\hline \multirow{4}{*}{$\begin{array}{l}\text { Terapia da Fala } \\
\mathrm{n}=23\end{array}$} & Todas & & 7 & 1 & 2 & $10(44 \%)$ \\
\hline & Nenhuma (por rotina) & 1 & 2 & 1 & & $4(17 \%)$ \\
\hline & $\begin{array}{l}\text { Se DA de determinada } \\
\text { gravidade (qual) }\end{array}$ & & $\begin{array}{c}2 \\
\text { (DA } \geq \text { moderado) }\end{array}$ & $\begin{array}{c}4 \\
\text { (DA } \geq \text { moderado) }\end{array}$ & & $6(26 \%)$ \\
\hline & "Não sei responder $(\ldots)$ " & & & 3 & & $3(13 \%)$ \\
\hline Total & & & & & & 23 \\
\hline \multirow{4}{*}{$\begin{array}{l}\text { Oftalmologia } \\
n=23\end{array}$} & Todas & & & 4 & & $4(17 \%)$ \\
\hline & Nenhuma (por rotina) & 1 & 7 & 3 & 2 & $13(57 \%)$ \\
\hline & $\begin{array}{l}\text { Se DA de determinada } \\
\text { gravidade (qual) }\end{array}$ & $\begin{array}{c}1 \\
(\mathrm{DA} \geq \text { severo })\end{array}$ & & & & $1(4 \%)$ \\
\hline & "Não sei responder $(\ldots)$ " & & 2 & 3 & & $5(22 \%)$ \\
\hline Total & & & & & & 23 \\
\hline \multirow{4}{*}{$\begin{array}{l}\text { Genética } \\
n=23\end{array}$} & Todas & & 1 & 2 & 2 & $5(22 \%)$ \\
\hline & Nenhuma (por rotina) & & 1 & 5 & & $6(26 \%)$ \\
\hline & $\begin{array}{l}\text { Se DA de determinada } \\
\text { gravidade (qual) }\end{array}$ & $\begin{array}{c}1 \\
(\mathrm{DA} \geq \mathrm{severo})\end{array}$ & $\begin{array}{c}4 \\
\text { (3 DA } \geq \text { moderado; } \\
1 \mathrm{DA} \geq \text { severo) }\end{array}$ & $\begin{array}{c}3 \\
\text { (2 DA } \geq \text { moderado; } \\
1 \text { DA } \geq \text { severo) }\end{array}$ & & $8(35 \%)$ \\
\hline & "Não sei responder (...)" & & 1 & 3 & & $4(17 \%)$ \\
\hline Total & & & & & & 23 \\
\hline \multirow{3}{*}{$\begin{array}{l}\text { SNIP } \\
n=23\end{array}$} & Todas & & 3 & 3 & 1 & $7(30 \%)$ \\
\hline & Raramente & & 1 & 1 & 1 & $3(13 \%)$ \\
\hline & "Não sei responder (...)" & 1 & 7 & 5 & & $13(57 \%)$ \\
\hline Total & & & & & & 23 \\
\hline
\end{tabular}

DA: défice auditivo; ES: entidade de saúde; H: hospital; ORL: Otorrinolaringologia; SC/SPA: surdez congénita/surdez precocemente adquirida; SNIPI: Sistema Nacional de Intervenção Precoce na Infância.

ORL A: Realiza o seguimento de todas as crianças com DA até à colocação do implante coclear (inclusive); ORL B: Realiza o seguimento de todas as crianças com DA, exceto aquelas que têm indicação para colocação de implante coclear; ORL C: Desconhece-se o seguimento que realiza † 1 das entidades de saúde é uma maternidade 
- Das 23 entidades de saúde 18 responderam que quando o SNIPI não é ativado as crianças são seguidas em diferentes consultas: Terapia da Fala $(n=15)$, Neuropediatria $(n=3)$, Pediatria do Desenvolvimento $(n=15)$, Pediatria $(n=4)$ e ainda pelo Serviço Social $(n=4)$.

- Nas restantes cinco entidades de saúde não é possível sabermos qual o apoio que é dado às crianças com SC/ SPA que não são referenciadas para o SNIPI, pois os serviços de Pediatria/ORL ou ambos os serviços de Pediatria e de ORL destes locais responderam "O meu serviço não é geralmente o responsável por essa referenciação".

3.4 Seguimento da criança com DA com indicação para implante coclear, além da ORL

Entidades de saúde representadas: 23 (as mesmas do ponto 3.2)

- 15 entidades de saúde (dois hospitais nível 3 e 13 hospitais nível $1 / 2$, onde se inclui um hospital que realiza o seguimento das crianças com indicação para colocação de implante coclear), referiram que estas crianças são seguidas em diferentes consultas: Terapia da Fala $(n=15)$, Pediatria do Desenvolvimento $(n=12)$, Neuropediatria $(n=4)$, Psicologia $(n=15)$.

- Oito entidades de saúde (três hospitais nível 1/2 que seguem as crianças com SC/SPA sem indicação para implante coclear e cinco cuja diferenciação no seguimento realizado às crianças com SC/SPA é desconhecido)

\section{DISCUSSÃO}

Este estudo multicêntrico mostra a realidade de 30 entidades de saúde ( $83 \%$ dos hospitais com maternidade/ maternidades do SNS) no âmbito da realização do RANU (quando o fazem e quem o realiza), do seguimento do RN REFERE na primeira fase do RANU e do RN com fatores de risco de surdez. Destas 30 entidades de saúde, 27 responderam à questão relativa ao número de nados-vivos nascidos no seu hospital/maternidade no ano de 2014 (40 196 nados-vivos) e 26 relativamente ao ano de 2015 (40 720 nados-vivos). A percentagem de nados-vivos nessas entidades de saúde face à natalidade nacional (Portugal continental e Ilhas) nesses dois anos foi de 48,8\% no ano de 2014 e de $47,6 \%$ em 2015. ${ }^{38}$ Estes valores permitem-nos dizer que a realidade destas 30 unidades de saúde afeta cerca de metade dos nados-vivos nascidos em Portugal. Nestas 30 entidades de saúde o RANU é realizado, por rotina, antes da alta hospitalar. Trata-se de uma realidade que contrasta com os resultados obtidos em estudos retrospetivos sobre dados de hospitais nível 1/2 publicados recentemente, que mostraram que a taxa de efetividade deste rastreio se aproxima, mas não atinge os $100 \% .^{39,40}$ Esse valor importa, uma vez que cada percentagem perdida corresponde a RN não rastreado. No questionário utilizado no nosso estudo perguntamos se o RANU é realizado 'por rotina' a todos os RN antes da alta. De facto, a resposta mostra o que é feito 'por rotina' nestas 30 entidades de saúde. Não podemos inferir através destas respostas que o RANU tem uma taxa de efetividade de $100 \%$ nestas entidades de saúde. Verificou-se que na maioria destas entidades de saúde o RANU é realizado por audiologistas, facto encontrado em outro estudo de âmbito nacional. ${ }^{36}$ As 30 entidades de saúde encaminham, sempre, para a segunda fase do rastreio/ ORL todas as crianças que não passam na primeira fase. O encaminhamento para ORL das crianças com fatores de risco de surdez, mesmo que passem na primeira fase do RANU, para se dar início ao acompanhamento audiológico especializado recomendado pelo GRISI, não é realizado em todas essas entidades de saúde. Isto significa que, em Portugal, nem todas as crianças com fator de risco de surdez são acompanhadas segundo as recomendações do GRISI e da DGS.

Os números de diagnósticos de SC/SPA revelados pelo conjunto de 15 entidades de saúde, 2,5 por cada 1000 em 2014 e 1,5 por cada 1000 em 2015, tal como a percentagem dos diferentes tipos de DA diagnosticados, foram idênticos aos encontrados noutros estudos e mostram a indiscutível importância deste rastreio. ${ }^{2,16}$ Importa referir que o número de nascimentos indicados por estas entidades de saúde em cada um dos anos (2014 e 2015) correspondem a $1 / 3$ dos nascimentos registados em Portugal. ${ }^{38}$

Quanto ao seguimento das crianças com SC/SPA, o número de entidades de saúde que cumpriram os critérios para serem incluídas nesta parte do estudo foi de 23 , que correspondem a $63 \%$ dos hospitais com maternidade/ maternidades do SNS. Os resultados mostraram que a avaliação/acompanhamento das crianças com SC/SPA por outras especialidades, além da ORL, varia consoante a entidade de saúde onde são seguidas (independentemente de se tratar de um hospital nível 1/2 ou 3). A avaliação por Pediatria/subespecialidade não é pedida, por rotina, para todas as crianças com SC/SPA em $50 \%$ das entidades. Em $27 \%$ das entidades, crianças com níveis mais graves de DA são sempre encaminhadas. Considerando a elevada prevalência de outras comorbilidades nesta população de doentes, nomeadamente patologia do neurodesenvolvimento, ${ }^{20,22}$ importa refletir se o encaminhamento para Pediatria/ subespecialidade, se deve realizar por rotina nas crianças com SC/SPA ou apenas se existir suspeita de alterações do desenvolvimento. No que diz respeito à referenciação para Terapia da Fala, esta realiza-se, por rotina, para todas as crianças com SC/SPA em $44 \%$ das entidades de saúde. Em $26 \%$ das entidades de saúde crianças com DA mais graves são referenciadas, por rotina, sendo que as crianças com DA ligeiro tendem a ser as menos encaminhadas. A elevada prevalência de atraso de desenvolvimento da linguagem mesmo em crianças com DA ligeiro impõe que se reflita sobre o modelo a seguir a respeito da referenciação destas crianças para Terapia da Fala. ${ }^{16,21}$ No que se refere ao encaminhamento para Oftalmologia, os resultados mostraram que esta não se realiza por rotina para todas as crianças com SC/SPA em $57 \%$ das entidades de saúde. Não foi possível saber a opção tomada em relação a este seguimento em $22 \%$ das entidades, uma vez que a resposta a esta questão foi "Não sei responder. O meu serviço não é geralmente o responsável por essa referenciação". Uma vez que as patologias oftalmológicas são muito frequentes 
nesta população de doentes e que o atraso na sua intervenção pode prejudicar a integração destas crianças na comunidade, que acumulam deste modo pelo menos dois défices sensitivos (auditivo e visual), importa reavaliar se devem ser encaminhadas todas as crianças ou apenas algumas e com que idades. ${ }^{41,42}$

Quanto ao encaminhamento para Genética das crianças com SC/SPA, de etiologia desconhecida, realiza-se, por rotina, em cerca de um quarto das entidades de saúde. As crianças com DA ligeiro a moderado são as menos referenciadas. Estes resultados contrastam com o facto de mais de $50 \%$ dos casos de surdez sem etiologia conhecida ser de causa genética, destes cerca de $60 \%$ é não sindromática e, portanto, a criança, regra geral, não apresenta dismorfismos ou outras comorbilidades que levem ao pedido de avaliação por Genética. Adicionalmente, quer nas alterações sindromáticas quer nas não sindromáticas existem formas progressivas de DA, pelo que o encaminhamento preferencial apenas das crianças com DA mais graves pode não ser a melhor opção. ${ }^{43,44}$

No que diz respeito à referenciação para o SNIPI, $57 \%$ das entidades de saúde (inclui oito entidades onde o Serviço de ORL realiza o seguimento das crianças com SC/SPA) responderam "Não sei responder. O meu serviço não é geralmente o responsável (...)". Adicionalmente, em todos os locais em que o SNIPI é ativado, quem o faz é o serviço de Pediatria. Estes dados mostram que muitos serviços de ORL desconhecem o SNIPI.

O seguimento das crianças com DA com indicação para implante coclear parece cumprir o recomendado pela NOC. Não nos é possível explicar o fato de 12 hospitais nível 1/2 que não seguem as crianças com indicação para implante coclear terem respondido ao seguimento realizado por estas crianças. A ausência da explicação deste facto prende-se com as limitações do estudo, que quer para este resultado como para todos os outros, devem ser tidas em consideração. Trata-se de um estudo transversal, baseado nas respostas obtidas através de um questionário, respondido numa determinada data o que lhe confere limitações intrínsecas. Mostra uma realidade factual correspondente à data na qual o questionário foi respondido e cujas perguntas podem ser alvo de diferentes interpretações. Além disso, pelo fato de algumas maternidades e alguns hospitais nível $1 / 2$ referenciarem as crianças com alterações no RANU para hospitais que não responderam ao questionário levam a que haja alguma limitação na análise das respostas dadas por estas maternidades/hospitais quando a algumas perguntas respondem: "Não sei responder. O meu serviço não é geralmente o responsável por essa referenciação".

\section{CONCLUSÃO}

O RANU parece estar amplamente difundido no SNS. O envolvimento de várias especialidades no seguimento das crianças com SC/SPA merece ser alvo de reflexão e discussão nacional. Se, por um lado, não é desejável criar avaliações/consultas dispensáveis num SNS tão sobrecarregado, por outro lado importa que o seguimento das crianças com SC/SPA seja o mais eficiente possível. Uma norma de orientação clínica para o seguimento da criança com DA, sem indicação para colocação de implante coclear, pode ser a peça chave. A eficácia desta NOC será tanto maior quanto mais se especifique/objetive o papel das outras especialidades, além da ORL, na intervenção precoce (entendida de forma mais global e não no âmbito estritamente audiológico). Adicionalmente, seria necessária a verificação do cumprimento da mesma, com medidas de controlo de qualidade que se deviam estender ao próprio RANU. A criação de uma ferramenta de trabalho comum para inserção de dados demográficos e clínicos dos doentes com SC/SPA a nível nacional permitiria o rápido acesso a dados epidemiológicos, informação valiosa na criação de conhecimento sobre este grupo de doentes a respeito dos quais ainda muito nos falta conhecer.

\section{PROTECÇÃO DE PESSOAS E ANIMAIS}

Os autores declaram que os procedimentos seguidos estavam de acordo com os regulamentos estabelecidos pelos responsáveis da Comissão de Investigação Clínica e Ética e de acordo com a Declaração de Helsínquia da Associação Médica Mundial.

\section{CONFIDENCIALIDADE DOS DADOS}

Os autores declaram ter seguido os protocolos do seu centro de trabalho acerca da publicação de dados.

\section{CONFLITOS DE INTERESSE}

Os autores declaram não ter conflitos de interesses relacionados com o presente trabalho.

\section{FONTES DE FINANCIAMENTO}

Este trabalho não recebeu qualquer tipo de suporte financeiro de nenhuma entidade no domínio público ou privado.

\section{REFERÊNCIAS}

1. Gravel J, Berg A, Bradley M, Cacace A, Campbell D, Dalzell L, et al. New York State universal newborn hearing screening demonstration project: effects of screening protocol on inpatient outcome measures. Ear Hear. 2000;21:131-40.

2. De Capua B, Costantini D, Martufi C, Latini G, Gentile M, De Felice C. Universal neonatal hearing screening: the Siena (Italy) experience on 19,700 newborns. Early Hum Dev. 2007;83:601-6.

3. Flynn M, Austin N, Flynn TS, Ford R, Buckland L. Universal newborn hearing screening introduced to NICU infants in Canterbury province, New Zealand. N Z Med J. 2004;117:U1183

4. Lévêque M, Schmidt $P$, Leroux B, Danvin JB, Langagne $T$, Labrousse $M$, et al. Universal newborn hearing screening: a 27-month experience in the French region of Champagne-Ardenne. Acta Paediatr. 2007;96:1150-4.

5. Mehl AL, Thomson V. Newborn hearing screening: the great omission. Pediatrics. 1998;101:E4.

6. Nagapoornima P, Ramesh A, Srilakshmi, Rao S, Patricia PL, Gore M, et al. Universal hearing screening. Indian J Pediatr. 2007;74:545-9.

7. Grindle CR. Pediatric hearing loss. Pediatr Rev. 2014;35:456-63.

8. Gifford KA, Holmes MG, Bernstein HH. Hearing loss in children. Pediatr Rev. 2009;30:207-15 
9. Goderis J, De Leenheer E, Smets K, Van Hoecke H, Keymeulen A, Dhooge I. Hearing loss and congenital CMV infection: a systematic review. Pediatrics. 2014;134:972-82.

10. van Dommelen P, Verkerk PH, van Straaten HL; Dutch Neonatal Intensive CareUnit Neonatal Hearing Screening Working Group. Hearing loss by week of gestation and birth weight in very preterm neonates. J Pediatr. 2015;166:840-3.

11. Uptodate.com. Hearing loss in children: Etiology. [consultado 2018 dez 14]. Disponível em https://www.uptodate.com/contents/hearing-lossin-children-etiology?search=Hearing $\% 20$ loss $\% 20$ in $\% 20$ children: $\% 20$ Etiology\&source=search_result\&selectedTitle=1 150\&usage type $=$ default\&display_rank $=1$.

12. Toriello HV, Reardon W, Gorlin RJ. Hereditary hearing loss and its syndromes. Oxford: Oxford University Press; 2004.

13. Direção Geral de Saúde. Norma de Orientação Clínica Número 018/2015. Rastreio e tratamento da surdez com implantes cocleares em idade pediátrica. Lisboa: DGS; 2015.

14. Tierney CD, Brown PJ, Serwint JR. Development of children who have hearing impairment. Pediatr Rev. 2008;29:e72-3.

15. Ching TY, Dillon H, Button L, Seeto M, Van Buynder P, Marnane V, et al. Age at intervention for permanent hearing loss and 5-year language outcomes. Pediatrics. 2017;140:pii:e20164274.

16. Aminoff MJ, Boller F, Swaab DF. Handbook of clinical neurology. In: Fitzpatrick E. Neurocognitive development in congenitally deaf children. Oxford: Elsevier; 2015. p. 335-56.

17. Moeller MP. Early intervention and language development in children who are deaf and hard of hearing. Pediatrics. 2000;106:E43.

18. Gérard JM, Deggouj N, Hupin C, Buisson AL, Monteyne V, Lavis C, et al. Evolution of communication abilities after cochlear implantation in prelingually deaf children. Int J Pediatr Otorhinolaryngol. 2010;74:642-8.

19. Birman CS, Elliott EJ, Gibson WP. Pediatric cochlear implants: additional disabilities prevalence, risk factors, and effect on language outcomes. Otol Neurotol. 2012;33:1347-52.

20. Karltorp E, Löfkvist U, Lewensohn-Fuchs I, Lindström K, Westblad ME Fahnehjelm KT, et al. Impaired balance and neurodevelopmental disabilities among children with congenital cytomegalovirus infection. Acta Paediatr. 2014;103:1165-73.

21. Wiley S, Arjmand E, Jareenmeinzen-Derr, Dixon M. Findings from multidisciplinary evaluation of children with permanent hearing loss. Int J Pediatr Otorhinolaryngol. 2011;75:1040-4.

22. Blanchard $M$, Thierry $B$, Marlin $S$, Denoyelle $F$. Aspects génétiques de la surdité. Arch Pediatr. 2012;19:886-9.

23. Häkli S, Luotonen M, Bloigu R, Majamaa K, Sorri M. Childhood hearing impairment in northern Finland, etiology and additional disabilities. Int J Pediatr Otorhinolaryngol. 2014;78:1852-6.

24. Ostadimoghaddam $\mathrm{H}$, Mirhajian $\mathrm{H}$, Yekta A, Sobhani Rad D, Heravian J, Malekifar A, et al. Eye problems in children with hearing impairment. J Curr Ophthalmol. 2015;27:56-9.

25. Abah ER, Oladigbolu KK, Samaila E, Merali H, Ahmed AO, Abubakar TH. Ophthalmologic abnormalities among deaf students in Kaduna, Northern Nigeria. Ann Afr Med. 2011;10:29-33.

26. Nikolopoulos TP, Lioumi D, Stamataki S, O'Donoghue GM. Evidence- based overview of ophthalmic disorders in deaf children: a literature update. Otol Neurotol. 2006;27:S1-24.

27. Guardino CA. Identification and placement for deaf students with multiple disabilities: choosing the path less followed. Am Ann Deaf. 2008;153:55-64.

28. Glascoe FP. Screening for developmental and behavioral problems. Ment Retard Dev Disabil Res Rev. 2005;11:173-9.

29. Sand N, Silverstein M, Glascoe FP, Gupta VB, Tonniges TP, O'Connor KG. Pediatricians' reported practices regarding developmental screening: do guidelines work? Do they help? Pediatrics. 2005;116:174-9.

30. Mackrides PS, Ryherd SJ. Screening for developmental delay. Am Fam Physician. 2011;84:544-9.

31. Jackson RL, Ammerman SB, Trautwein BA. Deafness and diversity: early intervention. Am Ann Deaf. 2015;160:356-67.

32. Moeller MP, Carr G, Seaver L, Stredler-Brown A, Holzinger D. Best practices in family-centered early intervention for children who are deaf or hard of hearing: an international consensus statement. J Deaf Stud Deaf Educ. 2013;18:429-45.

33. Yang Y, Liu YH, Fu MF, Li CL, Wang LY, Wang Q, et al. Home-based early intervention on auditory and speech development in Mandarin-speaking deaf infants and toddlers with chronological aged 7-24 months. Chin Med J. 2015;128:2202-7.

34. Direção Geral de Saúde. c2018-05. [Acedido 2018 Jan 20]. Disponível em: https://www.dgs.pt/sistema-nacional-de-intervencao-precoce-nainfancia.aspx.

35. Grandori F. European Consensus Statement on Neonatal Hearing Screening. J Laryngol Otol. 1998;112:1219.

36. Grupo de Rastreio Auditivo Neonatal Universal. Recomendações para o rastreio neonatal universal (RANU). Acta Pediatr Port. 2007:38:209-14.

37. Santos AR. Rastreio auditivo neonatal universal em Portugal: ponto da Situação. Tese de mestrado. Coimbra: Instituto Politécnico de Coimbra: 21012.

38. Direção Geral de Saúde. i024591. [consultado 2019 mai 25] Disponível em: https://www.dgs.pt/portal-da-estatistica-da-saude.aspx.

39. Gabriel T, Martins E, Carvalho G, Fontes N, Ramos MJ, Peres M, et al. Rastreio auditivo neonatal em 17732 recém-Nascidos. Acta Pediatr Port. 2017;48:14-8.

40. Caselhos S, Teles RV, Mexedo A, Fernandes F. Rastreio auditivo neonatal universal. Cinco anos de experiência. Rev Port Otorrinolaringol Cirurgia Cérvico-facial. 2012;50:137-40.

41. Donahue SP, Arthur B, Neely DE, Arnold RW, Silbert D, Ruben JB; POS Vision Screening Committee. Guidelines for automated preschool vision screening: a 10-year, evidence-based update. J AAPOS. 2013;17:4-8.

42. Koning HJ, Groenewoud JH, Lantau VK, Tjiam AM, Hoogeveen WC, de Faber JT, et al. Effectiveness of screening for amblyopia and other eye disorders in a prospective birth cohort study. J Med Screen. 2013;20:66-72.

43. Faundes V, Pardo RA, Castillo Taucher S. Genética de la sordera congénita. Med Clin. 2012;139:446-51.

44. Koffler T, Ushakov K, Avraham KB. Genetics of hearing loss: syndromic. Otolaryngol Clin North Am. 2015;48:1041-61. 